\title{
Assessment of Angiographic Vascularity of Meningiomas with Dynamic Susceptibility Contrast-Enhanced Perfusion-Weighted Imaging and Diffusion Tensor Imaging
}

\author{
C.H. Toh, K.-C. Wei, C.N. Chang, Y.-W. Peng, S.-H. Ng, H.-F. Wong, and C.-P. Lin
}

\begin{abstract}
BACKGROUND AND PURPOSE: The roles of DTI and dynamic susceptibility contrast-enhanced-PWI in predicting the angiographic vascularity of meningiomas have not been studied. We aimed to investigate if these 2 techniques could reflect the angiographic vascularity of meningiomas.
\end{abstract}

MATERIALS AND METHODS: Thirty-two consecutive patients with meningiomas who had preoperative dynamic susceptibility contrastenhanced-PWI, DTI, and conventional angiography were retrospectively included. The correlations between angiographic vascularity of meningiomas, classified with a 4-point grading scale, and the clinical or imaging variables_age and sex of patient, as well as size, CBV, fractional anisotropy, and ADC of meningiomas - were analyzed. The meningiomas were dichotomized into high-vascularity and lowvascularity groups. The differences in clinical and imaging variables between the 2 groups were compared. Receiver operating characteristic curve analysis was used to determine the diagnostic performance of these variables.

RESULTS: In meningiomas, angiographic vascularity correlated positively with CBV but negatively with fractional anisotropy. High-vascularity meningiomas demonstrated significantly higher CBV but lower fractional anisotropy as compared with low-vascularity meningiomas. In differentiating between the 2 groups, the area under the curve values were 0.991 for CBV and 0.934 for fractional anisotropy on receiver operating characteristic curve analysis.

CONCLUSIONS: CBV and fractional anisotropy correlate well with angiographic vascularity of meningiomas. They may differentiate between low-vascularity and high-vascularity meningiomas.

ABBREVIATIONS: $A \cup C=$ area under the curve; $F A=$ fractional anisotropy; $R O C=$ receiver operating characteristic

M eningiomas account for approximately one-third of primary brain tumors. ${ }^{1}$ Preoperative evaluation of meningioma with conventional angiography, the reference standard for tumor vasculature assessment, may help in surgical planning by providing important information such as tumor vascularity, vascular anatomy of feeding arteries, and draining veins. However,

Received March 15, 2013; accepted after revision April 27.

From the Departments of Medical Imaging and Intervention (C.H.T, Y.-W.P., S.-H.N, H.-F.W.) and Neurosurgery (K.-C.W., C.N.C.), Chang Gung Memorial Hospital, Linkou and Chang Gung University College of Medicine, Tao-Yuan, Taiwan; and Department of Biomedical Imaging and Radiological Sciences (C.H.T., C.-P.L.) and Brain Connectivity Laboratory (C.H.T, C.-P.L.), Institute of Neuroscience, National YangMing University, Taipei, Taiwan.

This work was partly supported by grants from the National Science Council Taiwan (NSC-102-2314-B-182-055 to C.H.T.).

Please address correspondence to Cheng Hong Toh, MD, Department of Medical Imaging and Intervention, Chang Gung Memorial Hospital, Linkou and Chang Gung University College of Medicine, No. 5, Fuxing St, Guishan Township, Taoyuan County 333, Taiwan; e-mail: eldomtoh@hotmail.com

-- Indicates open access to non-subscribers at www.ajnr.org

http://dx.doi.org/10.3174/ajnr.A3651 cerebral conventional angiography is invasive and not without risk. A previous study reported that there were $1.3 \%$ neurologic complications, among which $0.5 \%$ were permanent. ${ }^{2}$

Several MR imaging techniques have been shown to be able to provide some of the vascular information of meningiomas that could only be obtained with conventional angiography in the past. Arterial spin-labeling and regional perfusion imaging techniques could determine if the vascular supply of a meningioma was from the external carotid artery, the ICA, or both. ${ }^{3}$ MRA, on the other hand, helped to identify the arterial branches primarily supplying the meningiomas. ${ }^{4}$ To our knowledge, there is no report on the use of quantitative MR techniques to predict the degree of angiographic vascularity of meningiomas.

In contrast to conventional MR imaging, which provides only structural information, advanced MR techniques such as dynamic susceptibility contrast-enhanced PWI and DTI may provide physiologic information that helps in lesion characterization. The attenuation of T2-weighted signal measured with DTI after 2 extra gradient pulses can be linked to water diffusivity. Fractional 
anisotropy (FA) and ADC are quantitative metrics derived from DTI for water diffusivity measurement. ${ }^{5}$ DSC-PWI, on the other hand, measures $\mathrm{T} 2{ }^{\star}$-weighted signal intensity loss that occurs dynamically over bolus injection of contrast medium, from which relative $\mathrm{CBV}$, a quantitative marker of tumor angiogenesis, can be computed. $^{6}$

Both DTI ${ }^{7,8}$ and DSC-PWI ${ }^{9-11}$ had been reported to be useful in subtyping meningiomas. The microvessel area of meningiomas determined by histopathology was found to correlate with relative CBV derived from DSC-PWI. ${ }^{11}$ In the present study, we aimed to investigate if DTI and DSC-PWI could reflect the angiographic vascularity of meningiomas. To our knowledge, the roles of DTI and DSC-PWI in assessing the angiographic vascularity of meningiomas have never been studied.

\section{MATERIALS AND METHODS \\ Patients}

Between 2009-2012, a total of 46 patients underwent surgery for intracranial meningiomas in our institution. A routine MR protocol including conventional MR imaging, DSC-PWI, and DTI has been used to assess all patients with intracranial mass lesions since 2009. In our institution, conventional angiography has been a routine preoperative study in patients with meningioma who do not have iodinated contrast medium allergy or renal insufficiency. Nine patients whose MR studies were performed at outside hospitals were excluded. Thirty-seven patients whose preoperative MR imaging and conventional angiography were performed in our institution were retrospectively included. Signed informed consent was obtained from all patients for imaging and surgical procedures performed. Approval for reviewing the patient clinical data, findings of preoperative MR imaging studies, and catheter cerebral angiography was obtained from the institutional review board. Images with motion artifacts from 2 patients were excluded. Three patients with purely calcified tumors as seen on SWI or noncontrast CT images were excluded. Therefore, a total of 32 patients ( 17 men, 15 women; mean age, 54.5 years; age range, $24-80$ years) with meningioma (mean size, $5.3 \pm 1.5 \mathrm{~cm}$; range, $2-8 \mathrm{~cm}$ ) were analyzed. Histologic diagnosis was obtained in all patients by surgical resection. The histologic subtypes included 11 meningothelial, 8 transitional, 4 fibroblastic, 2 psammomatous, 3 microcystic, 2 atypical, and 2 anaplastic meningiomas. None of the patients had begun corticosteroid treatment, radiation therapy, or chemotherapy or had any previous brain biopsy at the time of MR imaging. Patients with estimated glomerular filtration rate $<60 \mathrm{mg} / \mathrm{min}$ per $1.72 \mathrm{~m}^{2}$ were excluded before enrollment.

\section{MR Imaging}

All MR studies were performed by use of a 3T unit (Magnetom Tim Trio; Siemens, Erlangen, Germany) with a 12-channel phased-array head coil. The conventional MR pulse sequences included transverse T1WI, transverse T2WI, and transverse FLAIR. DTI was performed in the axial plane by use of single-shot EPI with the following parameters: TR ms/TE ms, 5800/83; diffusion gradient encoding in 20 directions; $b=0,1000$ seconds/ $\mathrm{mm}^{2}$; FOV , $256 \times 256 \mathrm{~mm}$; matrix size, $128 \times 128$; section thickness, $2 \mathrm{~mm}$; and number of signals acquired, 4 . A total of 50-60 sections without intersection gap were used to cover the cerebral hemispheres, upper brain stem, and cerebellum. Generalized autocalibrating partially parallel acquisitions (reduction factor $=2$ ) were used during DTI acquisitions.

The DSC-PWI was obtained with a $\mathrm{T} 2{ }^{*}$-weighted gradientecho EPI sequence during the bolus injection of a standard dose $(0.1 \mathrm{mmol} / \mathrm{kg})$ of intravenous gadopentetate dimeglumine (Magnevist; Schering, Berlin, Germany). The injection rate was 4 $\mathrm{mL} / \mathrm{s}$ for all patients and was immediately followed by a bolus injection of saline (total of $20 \mathrm{~mL}$ at the same rate). DSC-PWI sequence parameters included the following: TR/TE, 1640/40 ms; flip angle, $90^{\circ}$; FOV , $230 \times 230 \mathrm{~mm}$; section thickness, $4 \mathrm{~mm}$; 20 sections; and acquisition time of 1 minute, 28 seconds. Fifty measurements were acquired, allowing acquisition of at least 5 measurements before bolus arrival. No contrast agent was administered before DSC-PWI. Postcontrast magnetization-prepared rapid acquisition gradient echo (TR/TE, 2000/2.63 ms; section thickness, $1 \mathrm{~mm}$; TI, $900 \mathrm{~ms}$; acquisition matrix, $224 \times 256$; and FOV, $224 \times 256 \mathrm{~mm}$ ) sequences were acquired after completion of the PWI sequence.

Conventional angiography was performed by interventional neuroradiologists through the femoral approach. Biplanar intraarterial DSA was performed by selective catheterization of bilateral internal and external carotid arteries as well as bilateral vertebral arteries. Images were obtained with a $1024 \times 1024$ matrix and a $17-\mathrm{cm}$ FOV. The temporal resolution of the images was 3 frames per second. A bolus of 5-9 $\mathrm{mL}$ of undiluted iodinated contrast material was injected for each projection by use of a power injector.

\section{Image Postprocessing}

The perfusion and diffusion-tensor data were transferred to an independent workstation and processed by use of the software nordicICE (Version 2, NordicNeuroLab, Bergen, Norway). The diffusion-weighted images were co-registered to the non-diffusion-weighted $(b=0)$ images to minimize the artifacts induced by eddy-current and subject motion. FA and ADC were calculated from diffusion-tensor data by use of standard algorithms described previously. $5,12,13$

The CBV for each voxel was estimated by integrating the relaxivity-time curve converted from the dynamic signal intensity curve. Contrast leakage correction was performed by use of a technique outlined by Boxerman et al. ${ }^{14,15}$

\section{Image Analysis}

Two independent interventional neuroradiologists blinded to the DTI and DSC-PWI findings assessed the tumor vascularity by evaluating the entire series of angiographic images. On the basis of the attenuation of tumor stain, the degree of angiographic vascularity of meningioma is graded as the following: 0 indicated none; 1, minimal; 2, moderate; and 3, marked (Fig 1). For meningiomas with grade 0 or 1 vascularity, preoperative embolization or even diagnostic conventional angiography is considered not to be necessary. Meningiomas with grade 2 or 3 vascularity were candidates for embolization, provided that their feeders derived from the external carotid artery or dural branches that were safe to be embolized. Interobserver differences were resolved by consensus. 


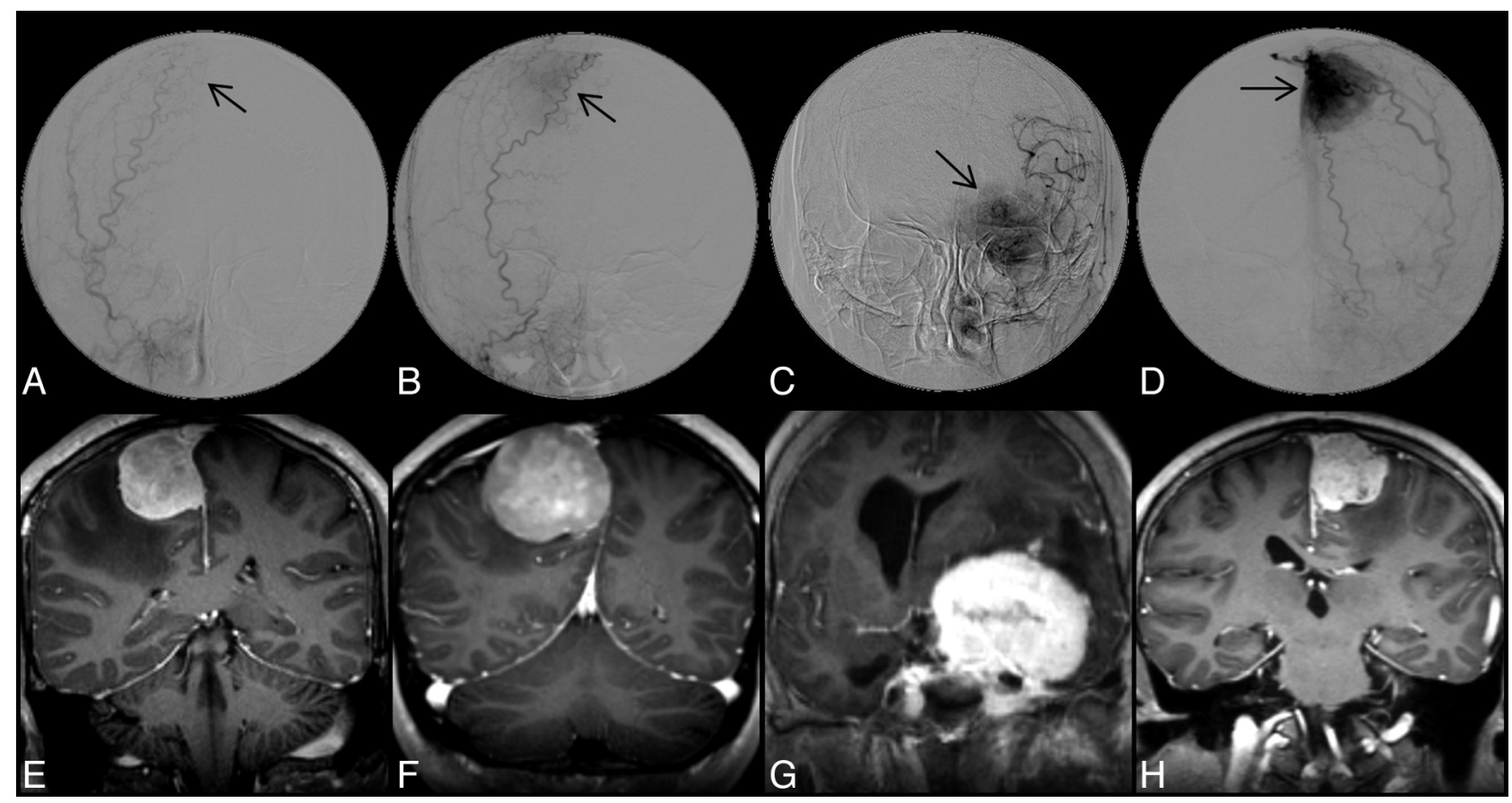

FIG 1. Upper panel shows DSA images of 4 different meningiomas, demonstrating grade $0(A), 1(B), 2(C)$, and $3(D)$ angiographic vascularity, respectively. Arrows indicate locations of meningiomas; lower panel shows the corresponding postcontrast MPRAGE images in coronal view.

A diagnostic neuroradiologist blinded to the angiographic results measured the size, ADC, FA, and CBV of the meningiomas. For tumor size, the largest diameter of the contrast-enhancing lesion on axial postcontrast MPRAGE was measured. Before all quantitative measurements, the ADC, FA, and CBV maps were coregistered to postcontrast MPRAGE on the basis of 3D nonrigid transformation and mutual information with the use of Statistical Parametric Mapping 2 (SPM2; Wellcome Department of Imaging Neuroscience, London, United Kingdom).

On the basis of postcontrast MPRAGE, a polygonal ROI was first drawn to include the entire enhancing lesion on every section. A threshold pixel value was then manually chosen to create a volumetric ROI to segment the entire enhancing tumor. The mean ADC, FA, and CBV of the whole tumor were subsequently measured with the tumor ROI. All ROIs did not include areas of necrosis or nontumor macrovessels evident on postcontrast MPRAGE. Examples of 2 meningiomas with grade 1 and grade 3 vascularity, respectively, are shown in Fig 2.

The ADC, FA, and CBV were normalized and expressed as ratios to contralateral normal-appearing white matter before all quantitative comparisons. The ratios were calculated by dividing the mean values of whole tumor by the values obtained from a region of interest (size range, $30-50 \mathrm{~mm}^{2}$ ) placed in the contralateral normal-appearing white matter.

\section{Statistical Analysis}

The level of interobserver agreement for angiographic vascularity was determined by calculating the $\kappa$ coefficient. The correlations between angiographic vascularity of meningiomas on the basis of consensus readings and the clinical or imaging variables - age and sex of patient, as well as size, CBV, FA, and ADC of meningiomas-were analyzed with the Spearman rank correlation coefficient.
The degree of angiographic tumor vascularity was further dichotomized into low-vascularity (grade 0 and 1 ) and high-vascularity (grade 2 and 3 ) groups. Between the 2 groups, the size, ADC, $\mathrm{FA}$, and CBV of meningiomas, as well as the patient age. were compared by means of a 2-sample $t$ test. The difference in sex was analyzed with $\chi^{2}$ analysis. The diagnostic performance of clinical and imaging variables with statistical significance was further determined by receiver operating characteristic (ROC) curve analysis. A commercially available statistical software package (SPSS 16; IBM, Armonk, New York) was used for analysis, and $P$ values $<.05$ were considered to indicate a statistically significant difference.

\section{RESULTS}

Interobserver agreement was excellent $(\kappa=0.824 ; P<.001)$ for degree of angiographic vascularity. The angiographic vascularity on consensus readings was grade 0 in 4 , grade 1 in 6 , grade 2 in 8 , and grade 3 in 14 meningiomas. Angiographic vascularity correlated positively with CBV (Spearman $\rho=0.891 ; P<.001$; Fig $3 A$ ) but negatively with FA (Spearman $\rho=-0.861 ; P<.001$; Fig 3B). There was no correlation between angiographic vascularity and the patient sex (Spearman $\rho=0.151 ; P=.410$ ), tumor size (Spearman $\rho=-0.186 ; P=.307)$, or ADC (Spearman $\rho=0.287$; $P=.111$; Fig 3C).

The clinical and imaging data of the meningiomas are summarized in Table 1 . There were 10 patients with low-vascularity meningiomas (grade 0 and 1 ) and 22 with high vascularity (grades 2 and 3). There were no significant differences in the sex, age, and tumor size between the 2 groups. The high-vascularity meningiomas demonstrated significantly higher CBV (Fig $4 A$ ) but lower FA (Fig $4 B$ ) as compared with low-vascularity meningiomas. The 2 groups showed no difference in their ADC values (Fig 4C). In differentiating between low- and high-vascularity meningiomas, the sensitivity and specificity were $90.9 \%$ and $100 \%$, respectively, 


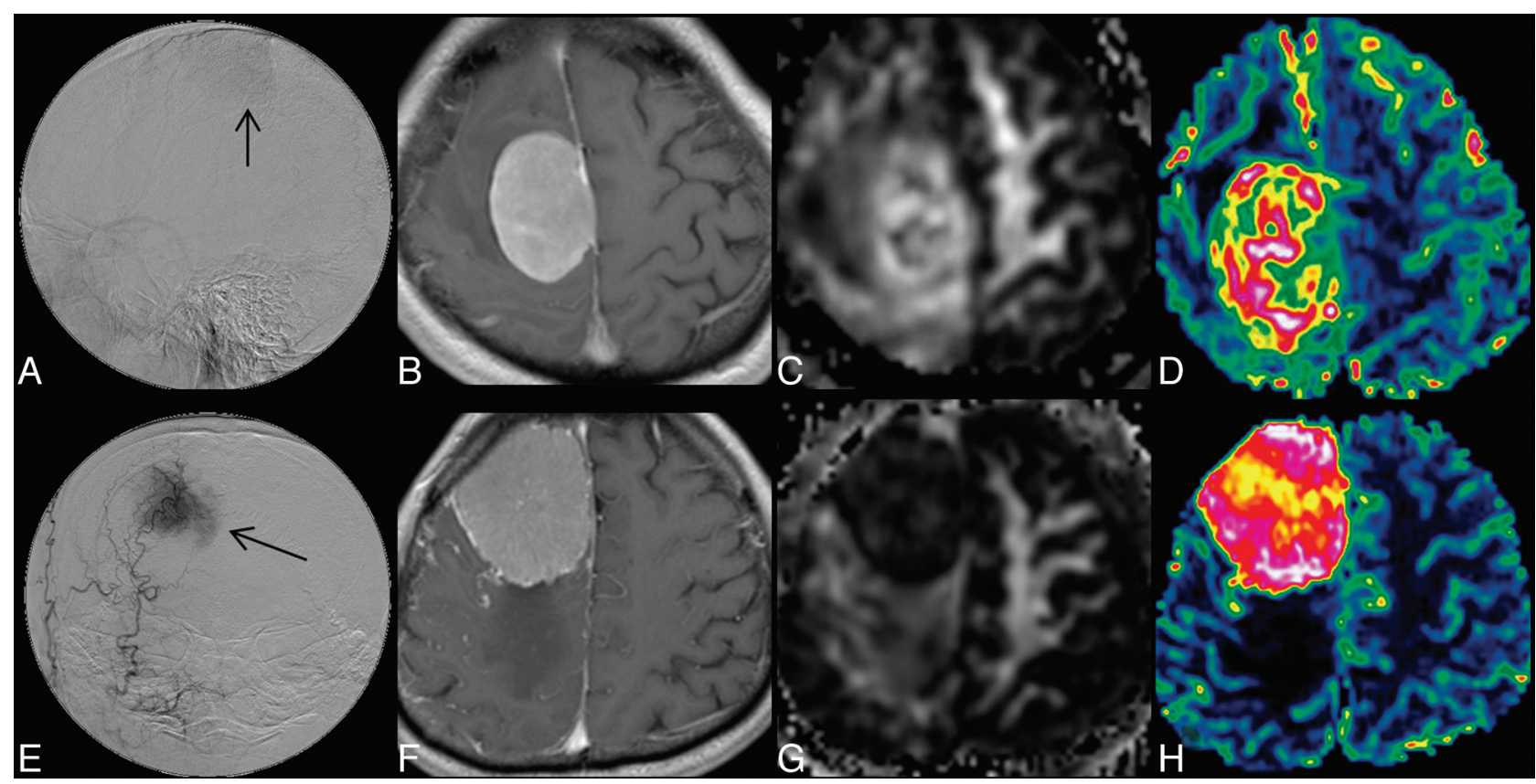

FIG 2. Upper panel shows DSA $(A)$, contrast-enhanced MPRAGE $(B)$, FA $(C)$, and CBV $(D)$ images of a meningioma with grade 1 angiographic vascularity; lower panel $(E-H)$ shows the corresponding images from a meningioma with grade 3 angiographic vascularity.

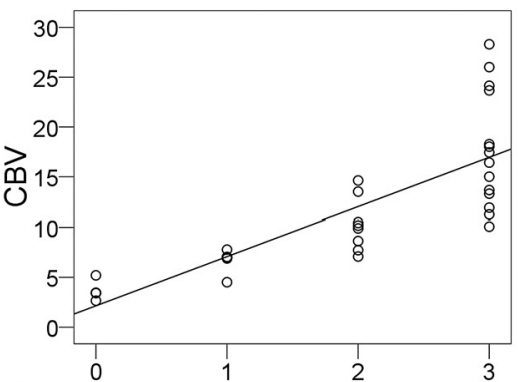

A Angiographic Vascularity Grade

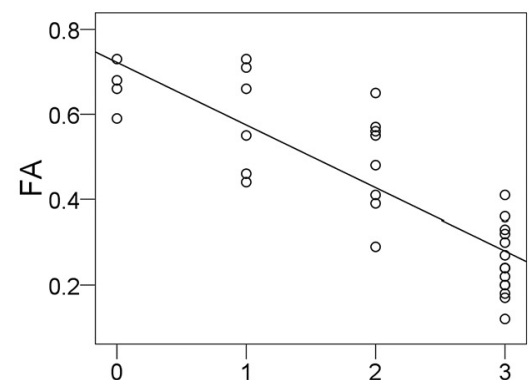

B Angiographic Vascularity Grade

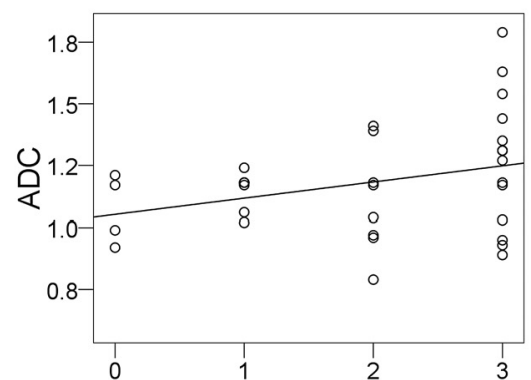

C Angiographic Vascularity Grade

FIG 3. Scatterplots with regression lines show a correlation (Spearman $\rho=0.891$ ) between CBV and angiographic vascularity $(A)$ and an inverse correlation (Spearman $\rho=-0.861$ ) between FA and angiographic vascularity $(B)$. There is no correlation between ADC and angiographic vascularity.

Table 1: Comparison of clinical and imaging data between low-vascularity and high-vascularity meningiomas

\begin{tabular}{lccrc}
\hline Group & $\begin{array}{c}\text { Low-Vascularity } \\
\text { Meningioma }\end{array}$ & $\begin{array}{c}\text { High-Vascularity } \\
\text { Meningioma }\end{array}$ & $\begin{array}{c}\boldsymbol{P} \\
\text { Value }\end{array}$ & \multicolumn{1}{c}{$95 \% \mathrm{Cl}$} \\
\hline Sex & 6 women, 4 men & 9 women, 13 men & .450 & NA \\
Age, y & $49.7 \pm 16.7$ & $56.7 \pm 14.5$ & .237 & -18.91 to 4.86 \\
Size, cm & $5.45 \pm 1.69$ & $5.27 \pm 1.57$ & .781 & -1.08 to 1.42 \\
CBV & $5.06 \pm 2.05$ & $13.97 \pm 5.67$ & $<.001$ & -12.71 to -5.10 \\
ADC & $1.114 \pm 0.107$ & $1.213 \pm 0.258$ & .253 & -0.27 to 0.07 \\
FA & $0.621 \pm 0.106$ & $0.339 \pm 0.147$ & $<.001$ & 0.17 to 0.38 \\
\hline
\end{tabular}

Note:-Data are mean \pm standard deviation. Units are $\times 10^{-3} \mathrm{~mm}^{2} / \mathrm{s}$ for ADC values.

for $\mathrm{CBV}$ and were $100 \%$ and $77 \%$, respectively, for FA. When combining CBV and FA, the sensitivity and specificity were $95.2 \%$ and $100 \%$, respectively. The results of ROC analysis are summarized in Table 2 and illustrated in Fig 5.

\section{DISCUSSION}

Our study showed that angiographic vascularity of meningiomas correlated with tumoral CBV and FA. Low-vascularity meningiomas demonstrated significantly lower CBV but higher FA when compared with high-vascularity meningiomas. Our results suggest that $\mathrm{CBV}$ and $\mathrm{FA}$ of meningiomas could reflect angiographic vascularity of the tumors.

Preoperative angiography evaluation and embolization of meningiomas is currently performed in some institutions, even though its value has not been established by randomized trials. ${ }^{16-18}$ Two very recent studies reported that patients may benefit from preoperative meningioma embolization. Shah et $\mathrm{al}^{17}$ reviewed 36 studies comprising 459 patients published between 1990-2011; they concluded that embolization may reduce rates of surgical morbidity and mortality in the management of meningiomas. In another study, Borg et $\mathrm{al}^{18}$ reported that complete devascularization resulted in lower blood transfusion requirements in their 107 patients with meningioma operated on between 2001$2010 .{ }^{18}$ We found that DSC-PWI and DTI could provide quantitative information about angiographic vascularity in a noninvasive way. Angiographic vascularity, which visualized as tumor stain, aids tumor localization during angiographic procedures and frequently serves as a reference of the degree of devascular- 

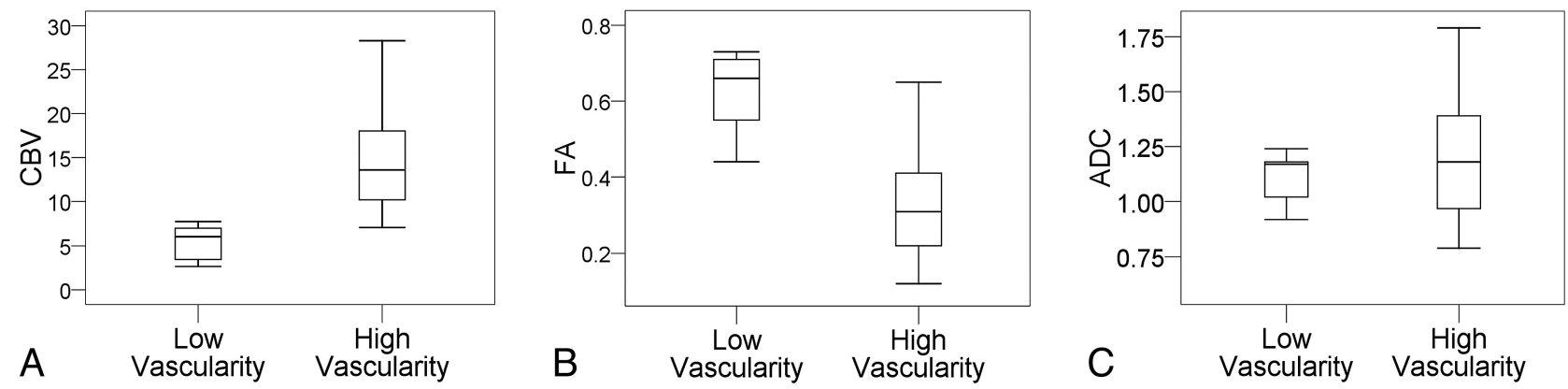

FIG 4. Boxplots of $C B V(A), F A(B)$, and $A D C(C)$ according to angiographic vascularity.

\begin{tabular}{|c|c|c|c|c|c|c|}
\hline Parameter & AUC & $95 \% \mathrm{Cl}$ & $P$ Value & CV & SEN & SPE \\
\hline CBV & 0.991 & $0.967-1.015$ & $<.001$ & 8.21 & 90.9 & 100 \\
\hline FA & 0.934 & $0.852-1.016$ & $<.001$ & 0.425 & 100 & 77 \\
\hline
\end{tabular}

Note:-CV indicates cutoff value; SEN, sensitivity; SPE, specificity. Data of sensitivity, specificity, and accuracy are in percentages.

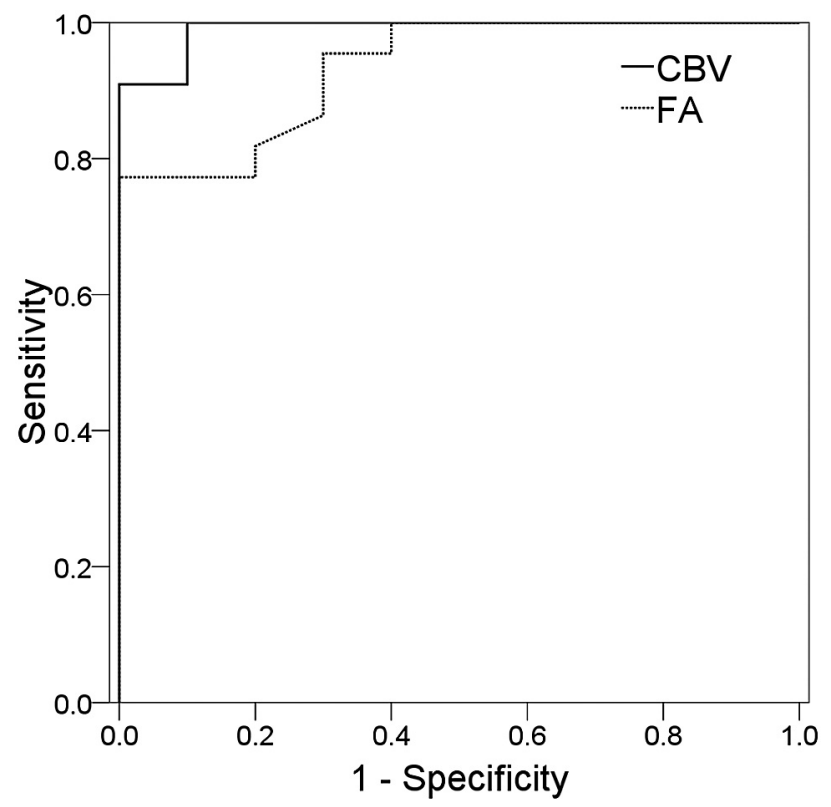

FIG 5. ROC curve analysis of the diagnostic performance of CBV and FA in differentiating between low-vascularity and high-vascularity meningiomas.

ization. Therefore, findings in our study may be helpful to institutions in which preoperative conventional angiography evaluation and embolization of meningiomas is practiced.

In the present study, all meningiomas demonstrated increased $\mathrm{CBV}$, a finding similar to what has been reported in the literature. ${ }^{10}$ However, not all meningiomas with increased CBV demonstrated tumor stain visible on conventional angiography. Rather, angiographic vascularity was absent in (grade 0) $12.5 \%$ of meningiomas and only slightly increased (grade 1) in $18.8 \%$ of cases. If a meningioma is identified to be low or absent of angiographic vascularity by DSC-PWI or DTI, embolization or even conventional angiography may not be needed because tumor feeders are frequently absent or difficult to identify. In contrast, if a meningioma is found to have high angiographic vascularity by
DSC-PWI or DTI, tumor embolization may be planned, and its associated risk and benefit can be discussed in advance with the patient.

CBV has been shown to be a surrogate marker of angiogenesis in gliomas. It has been found to correlate with microvascular proliferation ${ }^{19-22}$ and vascular endothelial growth factor expression, a major regulator of tumor angiogenesis. ${ }^{23}$ In meningiomas, both angiographic vascularity ${ }^{24}$ and $\mathrm{CBV}^{25}$ correlated with vascular endothelial growth factor expression. Therefore, it is not surprising when there is a positive correlation between CBV and angiographic vascularity in meningiomas, as demonstrated in the present study. To our knowledge, such correlation has not been reported in meningiomas, though it was observed in gliomas. ${ }^{26}$

The inverse correlation between FA and angiographic vascularity, as demonstrated in the present study, had allowed the use of FA to differentiate meningiomas of different angiographic vascularity, with diagnostic performance close to that of DSC-PWI. Therefore, DTI may serve as an alternative to DSC-PWI when administration of gadolinium-based contrast medium is a contraindication in patients who are subject to development of nephrogenic systemic fibrosis caused by low glomerular filtration rate. Previous studies investigated the roles of ADC and FA in subtyping meningiomas, but the results were controversial. ${ }^{7,8,27}$ In the present study, we found that angiographic vascularity of meningiomas correlated with FA but not with ADC. Whereas ADC measures the average changes of water diffusivity, FA quantifies the diffusion anisotropy. High FA indicates coherent diffusion, whereas low FA suggests disorganized or incoherent diffusion. The inverse correlation between FA and angiographic vascularity suggested that water diffusion in meningiomas became more disorganized as the angiographic vascularity increased.

It has been proposed that both pure diffusion of water molecules and microcirculation of the blood in the capillary network (perfusion-related diffusion) contribute to the signal decay observed on the source image of DTI. ${ }^{28}$ The perfusion-related diffusion can be considered as an incoherent motion caused by random capillary organization, and its contribution to the ADC measurement can be assessed by use of an intravoxel incoherent motion model. ${ }^{29,30}$ However, to our knowledge, there is no wellestablished model to evaluate the effect of perfusion on diffusion anisotropy. We speculate that higher perfusion resulted in greater incoherent motion and subsequently lower FA in meningiomas with high angiographic vascularity. On the other hand, the contribution of perfusion to the ADC measurement was limited at $b$ values $>100$ seconds $/ \mathrm{mm}^{2}{ }^{29}$ This, perhaps, may explain the ab- 
sence of correlation between angiographic vascularity and ADC obtained with a b value of 1000 seconds $/ \mathrm{mm}^{2}$ in the present study.

The purpose of preoperative evaluation with conventional angiography is to obtain information such as the degree of angiographic vascularity and the origin of arterial feeders of meningiomas. It has been reported that the information about vascular supply of meningiomas could be obtained with arterial spin-labeling, regional perfusion imaging, and MRA. ${ }^{3,4}$ Our results show that CBV and FA may complement arterial spin-labeling, regional perfusion imaging, and MRA by providing information about the degree of angiographic vascularity and may enhance the role of MR imaging in the preoperative assessment of meningiomas. Although the presence intratumoral vessels seen as flow voids on T2WI or enhancing vascular structures on postcontrast T1WI as well as the intensity of contrast enhancement may help to assess the vascularity of meningiomas, these imaging features cannot predict the angiographic vascularity of the tumor in a quantitative manner. In contrast, we have successfully demonstrated that CBV and FA could serve as quantitative markers to assess angiographic vascularity of meningiomas.

There are some limitations in the present study. First, there is no objective measurement of the degree of angiographic vascularity. Although the 4-point grading scale used in this study may be inherently subjective, it appears to be an optimal method, on the basis of its high interobserver agreement. Second, we did not have histologic findings to support the correlations between angiographic vascularity and FA or CBV. However, it is not a caveat to our study because we aimed to investigate the relationship between findings from different imaging modalities, for example, conventional angiography and MR imaging, and not between imaging findings and the pathologic changes. Although CBV and FA can predict angiographic vascularity of meningiomas, they cannot identify meningiomas with high angiographic vascularity but without accessible feeding arteries or meningiomas with a large arterial supply but with low angiographic vascularity. However, this limitation may be overcome in future studies if arterial spinlabeling, regional perfusion imaging and MRA are included in the MR protocol.

\section{CONCLUSIONS}

$\mathrm{CBV}$ and FA correlate well with angiographic vascularity of meningiomas. They may serve as noninvasive, quantitative tools to assess angiographic vascularity of meningiomas.

\section{ACKNOWLEDGMENTS}

The authors acknowledge support from Molecular Imaging Center, Chang Gung Memorial Hospital, Linkou, Taiwan.

Disclosures: Cheng Hong Toh—RELATED: Grant: National Science Council, Taiwan* ( ${ }^{*}$ money paid to institution).

\section{REFERENCES}

1. Wiemels J, Wrensch M, Claus EB. Epidemiology and etiology of meningioma. J Neurooncol 2010;99:307-14

2. Willinsky RA, Taylor SM, TerBrugge K, et al. Neurologic complications of cerebral angiography: prospective analysis of 2,899 procedures and review of the literature. Radiology 2003;227:522-28
3. Sasao A, Hirai T, Nishimura S, et al. Assessment of vascular supply of hypervascular extra-axial brain tumors with $3 \mathrm{~T}$ MR regional perfusion imaging. AJNR Am J Neuroradiol 2010;31:554-58

4. Uetani H, Akter M, Hirai T, et al. Can 3T MR angiography replace DSA for the identification of arteries feeding intracranial meningiomas? AJNR Am J Neuroradiol 2013;34:765-72

5. Mukherjee P, Berman JI, Chung SW, et al. Diffusion tensor MR imaging and fiber tractography: theoretic underpinnings. AJNR Am J Neuroradiol 2008;29:632-41

6. Zaharchuk G. Theoretical basis of hemodynamic MR imaging techniques to measure cerebral blood volume, cerebral blood flow, and permeability. AJNR Am J Neuroradiol 2007;28:1850-58

7. Toh CH, Castillo M, Wong AM, et al. Differentiation between classic and atypical meningiomas with use of diffusion tensor imaging. AJNR Am J Neuroradiol 2008;29:1630-35

8. Wang S, Kim S, Zhang Y, et al. Determination of grade and subtype of meningiomas by using histogram analysis of diffusion-tensor imaging metrics. Radiology 2012;262:584-92

9. Zhang H, Rodiger LA, Shen T, et al. Preoperative subtyping of meningiomas by perfusion MR imaging. Neuroradiology 2008; 50:835-40

10. Zhang H, Rodiger LA, Shen T, et al. Perfusion MR imaging for differentiation of benign and malignant meningiomas. Neuroradiology 2008;50:525-30

11. Kimura H, Takeuchi H, Koshimoto $\mathrm{Y}$, et al. Perfusion imaging of meningioma by using continuous arterial spin-labeling: comparison with dynamic susceptibility-weighted contrast-enhanced MR images and histopathologic features. AJNR Am J Neuroradiol 2006;27:85-93

12. Toh $\mathrm{CH}$, Wei $\mathrm{KC}, \mathrm{Ng} \mathrm{SH}$, et al. Differentiation of brain abscesses from necrotic glioblastomas and cystic metastatic brain tumors with diffusion tensor imaging. AJNR Am J Neuroradiol 2011;32:1646-51

13. Toh $\mathrm{CH}$, Wei KC, $\mathrm{Ng} \mathrm{SH}$, et al. Differentiation of tumefactive demyelinating lesions from high-grade gliomas with the use of diffusion tensor imaging. AJNR Am J Neuroradiol 2012;33:846-51

14. Boxerman JL, Schmainda KM, Weisskoff RM. Relative cerebral blood volume maps corrected for contrast agent extravasation significantly correlate with glioma tumor grade, whereas uncorrected maps do not. AJNR Am J Neuroradiol 2006;27:859-67

15. Toh $\mathrm{CH}$, Wei KC, Chang CN, et al. Differentiation of primary central nervous system lymphomas and glioblastomas: comparisons of diagnostic performance of dynamic susceptibility contrast-enhanced perfusion MR imaging without and with contrast-leakage correction. AJNR Am J Neuroradiol 2013;34:1145-49

16. Sluzewski M, van Rooij WJ, Lohle PN, et al. Embolization of meningiomas: comparison of safety between calibrated microspheres and polyvinyl-alcohol particles as embolic agents. AJNR Am J Neuroradiol 2013;34:727-29

17. Shah AH, Patel N, Raper DM, et al. The role of preoperative embolization for intracranial meningiomas. $J$ Neurosurg 2013;119:364-72

18. Borg A, Ekanayake J, Mair R, et al. Preoperative particle and glue embolization of meningiomas: indications, results and lessons learned from 117 consecutive patients. Neurosurgery 2013 Feb 25 [Epub ahead of print]

19. Hu LS, Eschbacher JM, Dueck AC, et al. Correlations between perfusion MR imaging cerebral blood volume, microvessel quantification, and clinical outcome using stereotactic analysis in recurrent high-grade glioma. AJNR Am J Neuroradiol 2012;33:69-76

20. Liao W, Liu Y, Wang X, et al. Differentiation of primary central nervous system lymphoma and high-grade glioma with dynamic susceptibility contrast-enhanced perfusion magnetic resonance imaging. Acta Radiol 2009;50:217-25

21. Sadeghi N, D'Haene N, Decaestecker C, et al. Apparent diffusion coefficient and cerebral blood volume in brain gliomas: relation to tumor cell density and tumor microvessel density based on stereotactic biopsies. AJNR Am J Neuroradiol 2008;29:476-82 
22. Barajas RF Jr, Phillips JJ, Parvataneni R, et al. Regional variation in histopathologic features of tumor specimens from treatment-naive glioblastoma correlates with anatomic and physiologic MR imaging. Neuro Oncol 2012;14:942-54

23. Maia AC Jr, Malheiros SM, da Rocha AJ, et al. MR cerebral blood volume maps correlated with vascular endothelial growth factor expression and tumor grade in nonenhancing gliomas. AJNR Am J Neuroradiol 2005;26:777-83

24. Bitzer M, Opitz H, Popp J, et al. Angiogenesis and brain oedema in intracranial meningiomas: influence of vascular endothelial growth factor. Acta Neurochir (Wien) 1998;140:333-40

25. Ginat DT, Mangla R, Yeaney G, et al. Correlation between dynamic contrast-enhanced perfusion MRI relative cerebral blood volume and vascular endothelial growth factor expression in meningiomas. Acad Radiol 2012;19:986-90

26. Sugahara T, Korogi Y, Kochi M, et al. Correlation of MR imaging- determined cerebral blood volume maps with histologic and angiographic determination of vascularity of gliomas. AJR Am J Roentgenol 1998;171:1479-86

27. Tropine A, Dellani PD, Glaser M, et al. Differentiation of fibroblastic meningiomas from other benign subtypes using diffusion tensor imaging. J Magn Reson Imaging 2007;25:703-08

28. Le Bihan $\mathrm{D}$, Breton $\mathrm{E}$, Lallemand $\mathrm{D}$, et al. MR imaging of intravoxel incoherent motions: application to diffusion and perfusion in neurologic disorders. Radiology 1986;161:401-07

29. Koh DM, Collins DJ, Orton MR. Intravoxel incoherent motion in body diffusion-weighted MRI: reality and challenges. $A J R A m \mathrm{~J}$ Roentgenol 2011;196:1351-61

30. Chavarria L, Alonso J, Garcia-Martinez R, et al. Biexponential analysis of diffusion-tensor imaging of the brain in patients with cirrhosis before and after liver transplantation. AJNR Am J Neuroradiol 2011;32:1510-17 